\title{
Body Mass Index and Hospital Mortality in Patients with Acute Coronary Syndrome Receiving Care in a University Hospital
}

\author{
Mercedes Camprubi, ${ }^{1}$ Sandra Cabrera, ${ }^{1}$ Jordi Sans, ${ }^{1}$ Georgina Vidal, ${ }^{2}$ \\ Teresa Salvadó, ${ }^{2}$ and Alfredo Bardají1 ${ }^{1}$ \\ ${ }^{1}$ Cardiology Service, Joan XXIII University Hospital of Tarragona IISPV, University Rovira Virgili, Calle Dr Mallafré Guash 4, \\ 43007 Tarragona, Spain \\ ${ }^{2}$ Education and Pathological Anatomy Units, Verge de la Cinta Hospital of Tortosa, Spain
}

Correspondence should be addressed to Alfredo Bardají, alfredo.bardaji@urv.cat

Received 27 January 2012; Revised 20 June 2012; Accepted 20 June 2012

Academic Editor: Jack A. Yanovski

Copyright (C) 2012 Mercedes Camprubi et al. This is an open access article distributed under the Creative Commons Attribution License, which permits unrestricted use, distribution, and reproduction in any medium, provided the original work is properly cited.

\begin{abstract}
Although obesity is a well-established cardiovascular risk factor, some controversy has arisen with regard to its effect on hospital mortality in patients admitted for acute coronary syndrome. Methods. Clinical and anthropometric variables were analyzed in patients consecutively admitted for acute coronary syndrome to a university hospital between 2009 and 2010, and the correlation of those variables with hospital mortality was examined. Results. A total of 824 patients with a diagnosis of myocardial infarction or unstable angina were analyzed. Body mass index was an independent factor in hospital mortality (odds ratio 0.739 (IC 95\%: $0.597-0.916), P=0.006)$. Mortality in normal weight $(n=218)$, overweight $(n=399)$, and obese $(n=172)$ subjects was $6.1 \%$, $3.1 \%$, and $4.1 \%$, respectively, with no statistically significant differences between the groups. Conclusions. There is something of a paradox in the relationship between body mass index and hospital mortality in patients with acute coronary syndrome in that the mortality rate decreases as body mass index increases. However, no statistically significant differences have been found in normal weight, overweight, or obese subjects.
\end{abstract}

\section{Introduction}

In addition to being a crucial underlying circumstance in the primary cardiovascular risk factors (CVRFs), obesity is an independent risk factor for cardiovascular illness and mortality $[1,2]$.

Increased body mass index (BMI) alters the behavior of adipose tissue, which provides insulin resistance as well as resistance to type-2 diabetes, arterial hypertension, dyslipidemia, and proinflammatory and prothrombotic states, thereby favoring the onset of ischemic cardiopathy [3]. An estimated $42.3 \%$ of coronary episodes in the Spanish population may be attributable to excess weight after adjusting for age, sex, and other risk factors [4].

It would therefore be logical to expect obesity to have a lethal effect on patients who have suffered a coronary event. However, some studies have reported better short- and medium-term prognoses in overweight coronary patients [5,
6]. This situation, in which obesity seems to protect patients with acute coronary syndrome (ACS), has been called "the obesity paradox" and has been described in other facilities. Other authors have obtained conflicting results and question the existence of this protective effect $[7,8]$.

In light of this controversy, the goal of this study is to determine the relationship between BMI and intrahospital mortality in patients admitted consecutively for ACS.

\section{Methods}

2.1. Patients and Study Design. All patients admitted consecutively between 2009 and 2010 for ACS were included in the RENACI database of the Working Group on Ischemic Heart Disease and Coronary Care Units of the Spanish Cardiology Society. Data were obtained of the clinical medical reports. A total of 853 patients were initially admitted during the period studied with a discharge diagnosis of unstable angina or 
myocardial infarction. No information on weight or size was available for 29 of the subjects. Therefore, the final sample consisted of 824 patients.

2.2. Variables and Events of Interest. The demographic characteristics and base anthropometry were recorded for all the patients studied. BMI was calculated as weight in kilograms divided by height in meters squared. BMI was studied as a quantitative and a categorical variable. The patients were divided into three groups: normal (BMI < $\left.25 \mathrm{~kg} / \mathrm{m}^{2}\right)$, overweight $\left(\mathrm{BMI}=25-30 \mathrm{~kg} / \mathrm{m}^{2}\right)$, and obese $\left(\right.$ BMI $\left.>30 \mathrm{~kg} / \mathrm{m}^{2}\right)$.

We analyzed various clinical variables including the classic CVRFs (smoking, diabetes, hypertension, and dyslipidemia), cardiovascular background and treatment prior to the acute event, hemodynamic variables at admission, risk scores according to the TIMI and GRACE scales, electrocardiographic data, analytical parameters and noninvasive cardiologic examination parameters, treatment administered at admission, coronarography examination, and results, as well as percutaneous and surgical revascularization.

Complications occurring during admission and mortality during hospital stays were also evaluated.

2.3. Statistical Analysis. All data were analyzed with the onesample Kolmogorov-Smirnov test to evaluate the normality of their distribution. Continuous variables were expressed as the average and standard deviation and were compared using an unpaired Student's $t$-test. The one-way ANOVA test was used to compare more than two groups, and the Bonferroni test was used to detect differences between groups. Percentages were compared using chi-squared statistics and Fisher's exact test when any of the expected values in any of the cells were below 10. The chi-squared test was used to compare more than two groups. Univariate and multivariate logistic regression analyses were used to determine the variables associated with hospital mortality.

\section{Results}

A total of 824 patients were studied. The average age of the sample was $65.84+0.4$ years (ranging from 25-95), and the sample was predominantly male $(73.5 \%)$. The mortality rate was $4.2 \%$ (35 patients).

3.1. BMI as a Quantitative Variable. BMI had an inverse relation to mortality, that is, to say, higher BMIs were associated with a reduced mortality rate. In the multivariate logistic regression analysis (Table 1), BMI along with other clinical variables (age, diabetes mellitus, GRACE score, and cardiogenic shock) were the independent predictors of death in our sample.

3.2. BMI Index as a Categorical Variable. Table 2 shows the hospital admission data for the sample, and Table 3 shows not only the distribution of subjects in the normal weight, overweight, and obese categories but also the main clinical variables (risk factors and relevant background factors).
TABLE 1: Multivariate logistic regression.

\begin{tabular}{lccc}
\hline Variable & $P$ & Odds ratio & IC 95\% \\
\hline Age & 0.003 & 1.122 & $1.041-1.208$ \\
Diabetes & 0.047 & 3.813 & $1.019-14.275$ \\
BMI & 0.006 & 0.739 & $0.597-0.916$ \\
GRACE score & 0.002 & 1.037 & $1.013-1.061$ \\
Killip IV admission & $<0.0001$ & 88.520 & $8.950-875.517$ \\
\hline
\end{tabular}

The incidence of risk factors is high in this population $(95.8 \%)$. In the obese group, the prevalence of diabetes mellitus and chronic obstructive pulmonary disease is significantly higher than in the other two groups. A larger proportion of the obese group had previously undergone treatment with angiotensin II receptor antagonists and diuretics although there were no other differences in prior treatments. At admission, overweight patients presented higher blood pressure levels, and lower Killip classes and TIMI scores, as well as higher initial glycemia and cholesterol levels. No significant differences were found in the ejection fraction of the three patient groups. A coronariography was conducted in $76 \%$ of the patients in our sample, and no differences were found between obese, overweight, or normal weight subjects. The incidence of coronary disease was similar across groups. No differences were found among the three groups in the percentage of angioplasties conducted or in the medical treatment received during admission. The prevalence of complications such as angina pectoris, reinfarction, hemorrhage, and mechanical complications was similar across groups with the exception of stroke, which was lower in obese patients. Thirty-five patients died (4.2\%) with no statistically significant differences among the different BMI groups. Most deaths occurred within 48 hours of having been admitted (19 patients), followed by 7 patients between day 2 and day 7, and a further 9 cases after day 7. No statistical differences were found in the combined endpoint (mortality, reinfarction, bleeding, and cerebrovascular accident).

\section{Discussion}

4.1. Key Findings. Our study shows that BMI is an independent predictor of hospital mortality in that a higher BMI is associated with a lower mortality rate. This finding is correlated with a lower mortality rate in obese and overweight patients compared to patients in the normal range although this result is not statistically significant.

4.2. Clinical Differences between Normal Weight, Overweight, and Obese Patients. Unlike other authors [5, 9], we did not find pronounced differences among these groups in terms of clinical data, hemodynamics, electrocardiographs, analyses, echocardiographs, angiographs, treatments administered, or resulting complications except that obese subjects have a greater incidence of diabetes, cardiovascular history, chronic obstructive pulmonary disease, and lower Killip classes and TIMI scores. This data suggests that evolution would be less 
TABle 2: Patients characteristics.

\begin{tabular}{|c|c|c|c|c|}
\hline & Normal weight & Overweight & Obese & $P$ \\
\hline Age & $65.4 \pm 14.6$ & $66.2 \pm 13$ & $65.2 \pm 11.9$ & 0.59 \\
\hline Heart rate & $75.2 \pm 18.4$ & $77.6 \pm 18$ & $78.7 \pm 18$ & 0.15 \\
\hline Blood pressure & $135 \pm 27$ & $141 \pm 32$ & $144 \pm 29$ & 0.005 \\
\hline KILLIP 3-4 & $12(5.3 \%)$ & $30(6.2 \%)$ & $14(7.8 \%)$ & 0.001 \\
\hline TIMI score & $3.71 \pm 1.46$ & $3.88 \pm 1.41$ & $4.07 \pm 1.33$ & 0.044 \\
\hline GRACE score & $130 \pm 41$ & $127 \pm 37$ & $124 \pm 35$ & 0.34 \\
\hline EKG ST depression & $43(18.9 \%)$ & $83(20 \%)$ & $38(21.1 \%)$ & 0.89 \\
\hline Elevated troponins & $198(86.8 \%)$ & $329(79.1 \%)$ & $151(83.9 \%)$ & 0.1 \\
\hline Creatinine & $1.15 \pm 0.75$ & $1.17 \pm 0.70$ & $1.11 \pm 0.63$ & 0.68 \\
\hline Cholesterol & $166 \pm 40$ & $175 \pm 43$ & $176 \pm 37$ & 0.024 \\
\hline Blood glucose levels & $145 \pm 68$ & $162 \pm 85$ & $173 \pm 89$ & 0.002 \\
\hline EF severely depressed & $16(7 \%)$ & $26(6.3 \%)$ & $16(8.9 \%)$ & 0.66 \\
\hline Cardiac catheterization & $174(76 \%)$ & $316(76 \%)$ & $141(78.3 \%)$ & 0.89 \\
\hline Two-vessel CD & $34(14.9 \%)$ & $85(20.4 \%)$ & $42(23.3 \%)$ & 0.09 \\
\hline Three-vessel CD & $18(7.9 \%)$ & $54(13 \%)$ & $26(14.4 \%)$ & 0.06 \\
\hline Coronary angioplasty & $124(54.4 \%)$ & $210(50.5 \%)$ & $89(49.4 \%)$ & 0.85 \\
\hline Postinfarction angina pectoris & $23(10.1 \%)$ & $37(8.9 \%)$ & $12(6.7 \%)$ & 0.47 \\
\hline Reinfarction & $12(5.3 \%)$ & $18(4.3 \%)$ & $8(4.4 \%)$ & 0.85 \\
\hline Cerebrovascular accident & $5(2.2 \%)$ & $1(0.2 \%)$ & $1(0.6 \%)$ & 0.03 \\
\hline Worst Killip during admission & $171(75 \%)$ & $341(82 \%)$ & $137(76.1 \%)$ & 0.03 \\
\hline Hemorrhage & $4(1 \%)$ & $9(2.1 \%)$ & $1(0.5 \%)$ & 0.24 \\
\hline Hospitalization length (days) & $6.8 \pm 6.2$ & $6.7 \pm 5.1$ & $6 \pm 3.2$ & 0.24 \\
\hline Hospital mortality & $14(6.1 \%)$ & $13(3.1 \%)$ & $8(4.1 \%)$ & 0.19 \\
\hline Combined endpoint & $24(11 \%)$ & $37(8.9 \%)$ & $17(9.4 \%)$ & 0.69 \\
\hline
\end{tabular}

No difference in antiplatelet, anticoagulant, inotrope, beta blocker, and diuretic treatment at admission. EF: ejection fraction, CD: coronary disease. Combined endpoint: mortality or reinfarction or bleeding or cerebrovascular accident.

favorable and the mortality rate higher during hospital stays. Paradoxically, this is not the case in our series.

4.3. The Obesity Paradox. Although BMI is an inverse predictor of mortality in our series, indicating that overweight patients with ACS have better survival rates than normal weight subjects, when subjects are classified into BMI subgroups (normal weight, overweight, and obese), we were unable to demonstrate with statistical significance that patients classified as obese have lower hospital mortality although this tendency does exist. Among other reasons, this may be due to the sample size. Our data is consistent with other data published [5], among which are the results of the SYNERGY [10], Merlin-TIMI 36 [6], and CRUSADE [9] studies, which also describe lower mortality rates in obese patients suffering from ACS. In a meta-analysis of 40 studies with more than 250,000 patients, Romero-Corral et al. [11] observed that overweight patients with coronary disease have a lower risk of cardiovascular and total mortality than patients in the low and normal weight groups. However, this tendency disappears in the $\mathrm{BMI} \geq 35 \mathrm{~kg} / \mathrm{m}^{2}$ patient group (morbid obesity), which runs a greater risk of cardiovascular mortality.

Other authors have not corroborated the protective effect of obesity in ACS [12]. Fiol et al. analyzed the prognostic value of BMI in medium-term hospital mortality in a cohort of 1,063 consecutive patients with first infarction in 15 hospitals in Spain [7] and found no association between BMI and medium-term hospital mortality. Furthermore, in patients with acute ST-elevated myocardial infarction, Das et al. [8] described a less-favorable prognosis for very obese patients in a sample of 501,489 patients.

Several hypotheses have been proposed to explain the inverse relationship between obesity and the prognosis of patients with ischemic cardiopathy. The higher mortality rate in the normal weight group may be due to a higher mortality rate in underweight patients. Some studies $[1,2]$ show that the optimal BMI for the general population is $22.5-25 \mathrm{~kg} / \mathrm{m}^{2}$ in nonsmokers and $24-27 \mathrm{~kg} / \mathrm{m}^{2}$ in smokers, and that people in the lower range of normal BMI (18.5$22.5 \mathrm{~kg} / \mathrm{m}^{2}$ ) have a higher mortality rate than individuals in the upper range of overweight BMI $\left(27.5-30 \mathrm{~kg} / \mathrm{m}^{2}\right)$. So the high-risk, underweight group is subsumed within the group of patients that is often considered normal. In contrast, most studies have found that a higher mortality rate is associated with extreme obesity $[8,9,11]$.

Another aspect that is often not given appropriate consideration is the role of pharmacological treatment, especially the adverse effects of multiple medicines used in ACS (fibrinolytic agents, antiplatelet drugs, anticoagulants, inotropes, antiarrhythmic agents, diuretics, nitrates, beta blockers, etc.), which could explain a higher mortality rate. 
TABLE 3: Risk factors and relevant history.

\begin{tabular}{|c|c|c|c|c|}
\hline & Normal weight & Overweight & Obese & $P$ \\
\hline \multicolumn{5}{|l|}{ Risk factors } \\
\hline Tobacco & $87(37.4 \%)$ & $136(32.9 \%)$ & $47(26.4 \%)$ & 0.009 \\
\hline Diabetes & $61(26.8 \%)$ & $143(34.4 \%)$ & $75(41.7 \%)$ & 0.016 \\
\hline Hypertension & $133(58.3 \%)$ & $280(67.3 \%)$ & $129(71.7 \%)$ & 0.063 \\
\hline Dyslipemia & $120(52.6 \%)$ & $247(59.4 \%)$ & $112(62.2 \%)$ & 0.24 \\
\hline CV history & $144(63.2 \%)$ & $275(66.1 \%)$ & $137(76.1 \%)$ & 0.015 \\
\hline Infarction & $44(19.3 \%)$ & $101(24.3 \%)$ & $51(28.3 \%)$ & 0.098 \\
\hline Heart failure & $13(5.7 \%)$ & $11(2.6 \%)$ & $10(5.6 \%)$ & 0.097 \\
\hline Peripheral vascular disease & $24(10.5 \%)$ & $36(8.7 \%)$ & $15(8.3 \%)$ & 0.67 \\
\hline Cerebrovascular accident & $16(7 \%)$ & $37(8.9 \%)$ & $9(5 \%)$ & 0.24 \\
\hline Renal failure & $23(10.1 \%)$ & $46(11.1 \%)$ & $16(8.9 \%)$ & 0.72 \\
\hline COPD & $22(9.6 \%)$ & $60(14.4 \%)$ & $34(18.9 \%)$ & 0.027 \\
\hline Prior PCI & $29(12.7 \%)$ & $46(11.1 \%)$ & $32(17.8 \%)$ & 0.2 \\
\hline Prior cardiac surgery & $11(4.8 \%)$ & $24(5.8 \%)$ & $7(3.9 \%)$ & 0.89 \\
\hline
\end{tabular}

No difference in treatment prior to admittance except greater use of ARA2 and diuretics in overweight and obese subjects than in normal subjects, CV: cardiovascular, COPD: chronic obstructive pulmonary disease, PCI: percutaneous coronary intervention.

Given the difficulty of anthropometric measurement in the first hours of cardiologic emergency treatment, errors in medicinal dosage may arise, primarily in those patients with lower body weight. Nevertheless, unlike other authors, in our series we found that BMI group had no effect on either treatments administered or hemorrhages [9].

4.4. Other Factors Related to BMI. Since BMI cannot differentiate between muscle and fat mass, overweight and obese subjects with coronary disease may have more muscle mass. When BMI is very high and better reflects body adiposity, the obesity paradox disappears [8].

The main approaches used to measure obesity are BMI, waist-hip ratio, and waist circumference. There is considerable disagreement on which of them is best $[3,13-$ 15]. Most authors recommend the simultaneous use of all of these parameters to better assess patient risk. Although the worst scenario is the obese individual with a high waist circumference, patients categorized as normal with a high waist circumference also face an elevated risk. Other authors suggest that obesity should be expressed as the percentage of body fat ( $>25$ for men and $>35$ for women), but this is difficult to quantify clinically. Correctly identifying the different compartments of body fat, and specifically visceral fat, using more sophisticated techniques $[16,17]$ may help to clarify the role of obesity in ACS patient mortality. Studies have also been conducted which measure the degree of activity or physical condition and classify the subgroups normal weight-sedentary, overweight active, obese active, overweight sedentary, and obese sedentary from least to greatest risk, with obese-sedentary being the group at greatest risk $[18,19]$.

4.5. Limitations of This Study. Although the sample consisted of more than 800 patients, classifying the patients into three groups may prevent hospital mortality from being appropriately assessed because its incidence is relatively low. The clinical appraisal of obesity has many limitations, and an alternative might be the combined appraisal of other anthropometric measurements such as waist circumference, which was an unknown variable in our sample. Better anthropometric appraisal using data on abdominal obesity and an understanding of the physical condition of the patients so that they can be more accurately classified into groups could play a critical role in clarifying this paradox.

\section{Conclusions}

The relationship between higher BMI and a greater incidence of coronary disease in the general population is well documented, and excess weight should clearly be avoided. However, once coronary heart disease arises, the association between BMI and the prognosis becomes more complex, even paradoxical according to some authors. So the controversy over the predictive value of BMI in patients with ACS remains latent. Our study confirms that BMI is an independent predictor of hospital mortality; a higher BMI is associated with a lower mortality rate. Our overweight and obese patients had a higher incidence of diabetes, cardiovascular history, chronic obstructive pulmonary disease, and lower Killip classes and TIMI scores. However, they did not show an increased mortality rate, which is apparently paradoxical.

\section{References}

[1] E. E. Calle, M. J. Thun, J. M. Petrelli, C. Rodriguez, and C. W. Heath Jr., "Body-mass index and mortality in a prospective cohort of U.S. adults," The New England Journal of Medicine, vol. 341, no. 15, pp. 1097-1105, 1999.

[2] G. Whitlock, S. Lewington, P. Sherliker et al., "Bodymass index and cause-specific mortality in 900,000 adults: 
collaborative analyses of 57 prospective studies," The Lancet, vol. 373, no. 9669, pp. 1083-1096, 2009.

[3] H. E. Bays, "Adiposopathy: is "sick fat" a cardiovascular disease?" Journal of the American College of Cardiology, vol. 57, no. 25, pp. 2461-2473, 2011.

[4] M. J. -Medrano, R. Pastor-Barriuso, R. Boix et al., "Riesgo coronario atribuible a los factores de riesgo cardiovascular en población española," Revista Española de Cardiología, vol. 60, pp. 1250-1256, 2007.

[5] H. J. Buettner, C. Mueller, M. Gick et al., "The impact of obesity on mortality in UA/non-ST-segment elevation myocardial infarction," European Heart Journal, vol. 28, no. 14, pp. 16941701, 2007.

[6] M. B. Kadakia, C. S. Fox, B. M. Scirica et al., "Central obesity and cardiovascular outcomes in patients with acute coronary syndrome: observations from the MERLIN-TIMI 36 trial," Heart, vol. 97, pp. 1782-1787, 2011.

[7] M. Fiol, R. Amezaga, R. Aros et al., "Indice de masa corporal como factor pronóstico en pacientes tras un primer infarto de miocardio," Medicina Clínica, vol. 135, no. 14, pp. 631-636, 2010.

[8] S. R. Das, K. P. Alexander, A. Y. Chen et al., "Impact of body weight and extreme obesity on the presentation, treatment, and in-hospital outcomes of 50, 149 patients with ST-segment elevation myocardial infarction: results from the NCDR (National Cardiovascular Data Registry)," Journal of the American College of Cardiology, vol. 58, pp. 2642-2650, 2011.

[9] D. B. Diercks, M. T. Roe, J. Mulgund et al., "The obesity paradox in non-ST-segment elevation acute coronary syndromes: results from the can rapid risk stratification of unstable angina patients suppress adverse outcomes with early implementation of the American College of Cardiology/American Heart Association guidelines quality improvement initiative," American Heart Journal, vol. 152, no. 1, pp. 140-148, 2006.

[10] K. W. Mahaffey, S. T. Tonev, S. A. Spinler et al., "Obesity in patients with non-ST-segment elevation acute coronary syndromes: results from the SYNERGY trial," International Journal of Cardiology, vol. 139, no. 2, pp. 123-133, 2010.

[11] A. Romero-Corral, V. M. Montori, V. K. Somers et al., "Association of bodyweight with total mortality and with cardiovascular events in coronary artery disease: a systematic review of cohort studies," The Lancet, vol. 368, no. 9536, pp. 666-678, 2006.

[12] M. Benderly, V. Boyko, and U. Goldbourt, "Relation of body mass index to mortality among men with coronary heart disease," American Journal of Cardiology, vol. 106, no. 3, pp. 297-304, 2010.

[13] K. M. Rexrode, V. J. Carey, C. H. Hennekens et al., "Abdominal adiposity and coronary heart disease in women," Journal of the American Medical Association, vol. 280, no. 21, pp. 1843-1848, 1998.

[14] S. Yusuf, S. Hawken, S. Ounpuu, T. Dans et al., "Effect of potentially modifiable risk factors associated with myocardial infarction in 52 countries(The INTERHEART Study): case control study," The Lancet, vol. 364, no. 9438, pp. 937-952, 2004.

[15] T. Coutinho, K. Goel, D. S. Corrêa et al., "Central obesity and survival in subjects with coronary artery disease: a systematic review of the literature and collaborative analysis with individual subject data," Journal of the American College of Cardiology, vol. 57, no. 19, pp. 1877-1886, 2011.

[16] G. Thanassoulis, R. S. Vasan, C. J. O'Donnell et al., "Distribution, and risk factor correlates of high pericardial and intrathoracic fat depots in the framingham heart study," Circulation, vol. 3, pp. 559-566, 2010.

[17] M. A. Cornier, J. P. Després, N. Davis et al., "Assessing adiposity: a scientific statement from the American Heart Association," Circulation, vol. 124, pp. 1996-2019, 2011.

[18] T. Y. Li, J. S. Rana, J. E. Manson et al., "Obesity as compared with physical activity in predicting risk of coronary heart disease in women," Circulation, vol. 113, no. 4, pp. 499-506, 2006.

[19] J. S. Rana, B. J. Arsenault, J. P. Després et al., "Inflammatory biomarkers, physical activity, waist circumference, and risk of future coronary heart disease in healthy men and women," European Heart Journal, vol. 32, no. 3, pp. 336-344, 2011. 


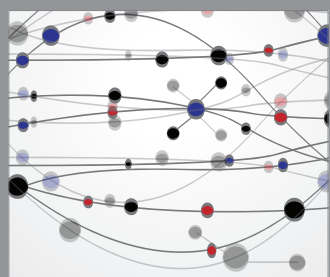

The Scientific World Journal
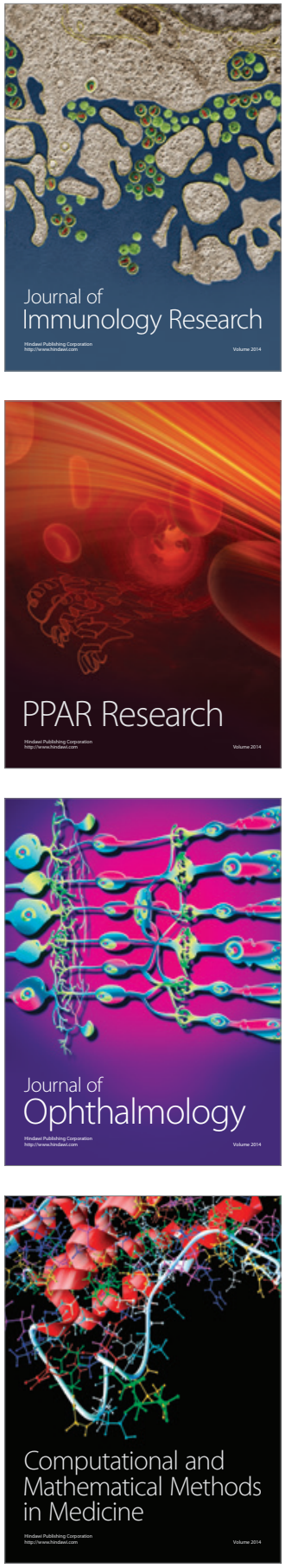

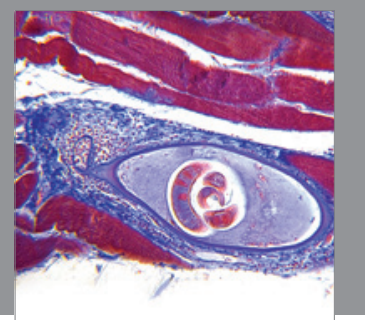

Gastroenterology

Research and Practice
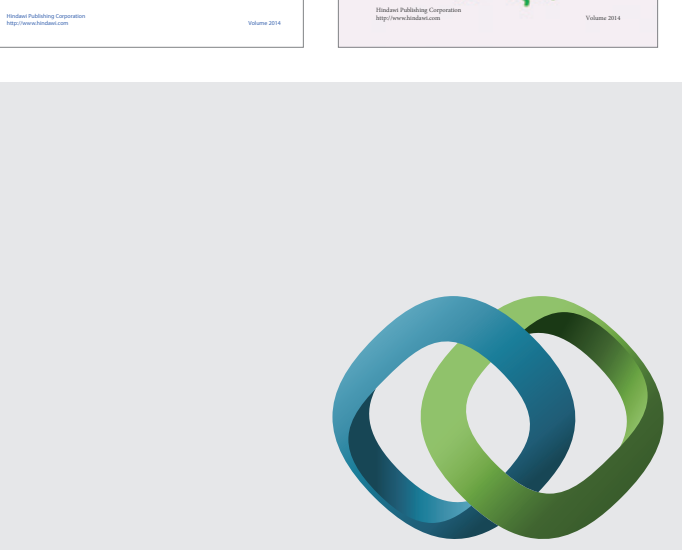

\section{Hindawi}

Submit your manuscripts at

http://www.hindawi.com
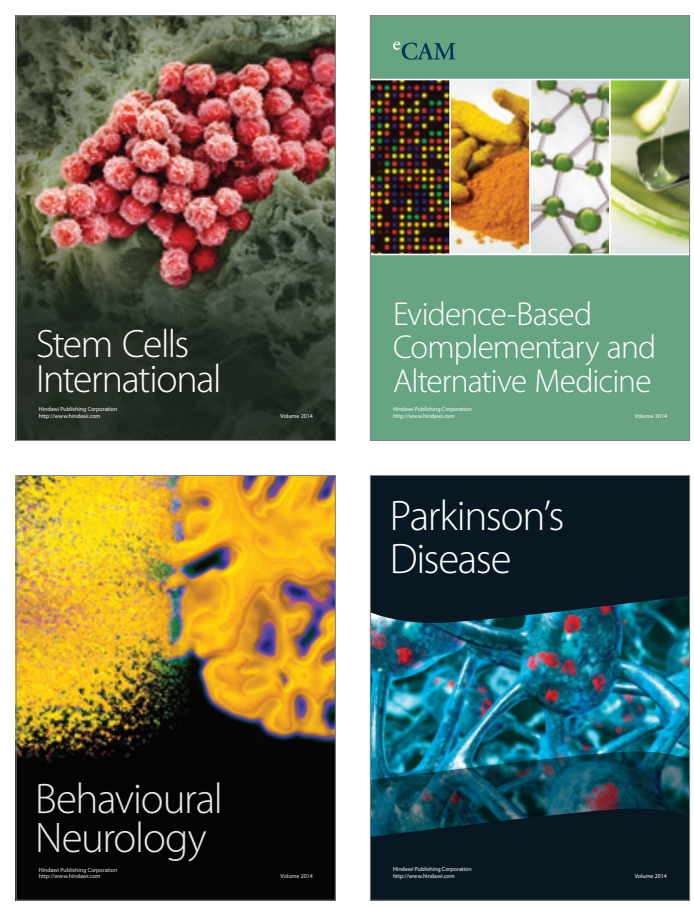

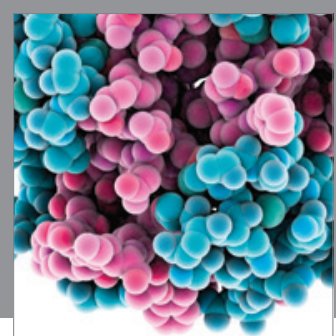

Journal of
Diabetes Research

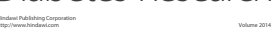

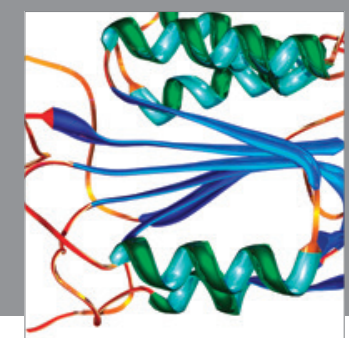

Disease Markers
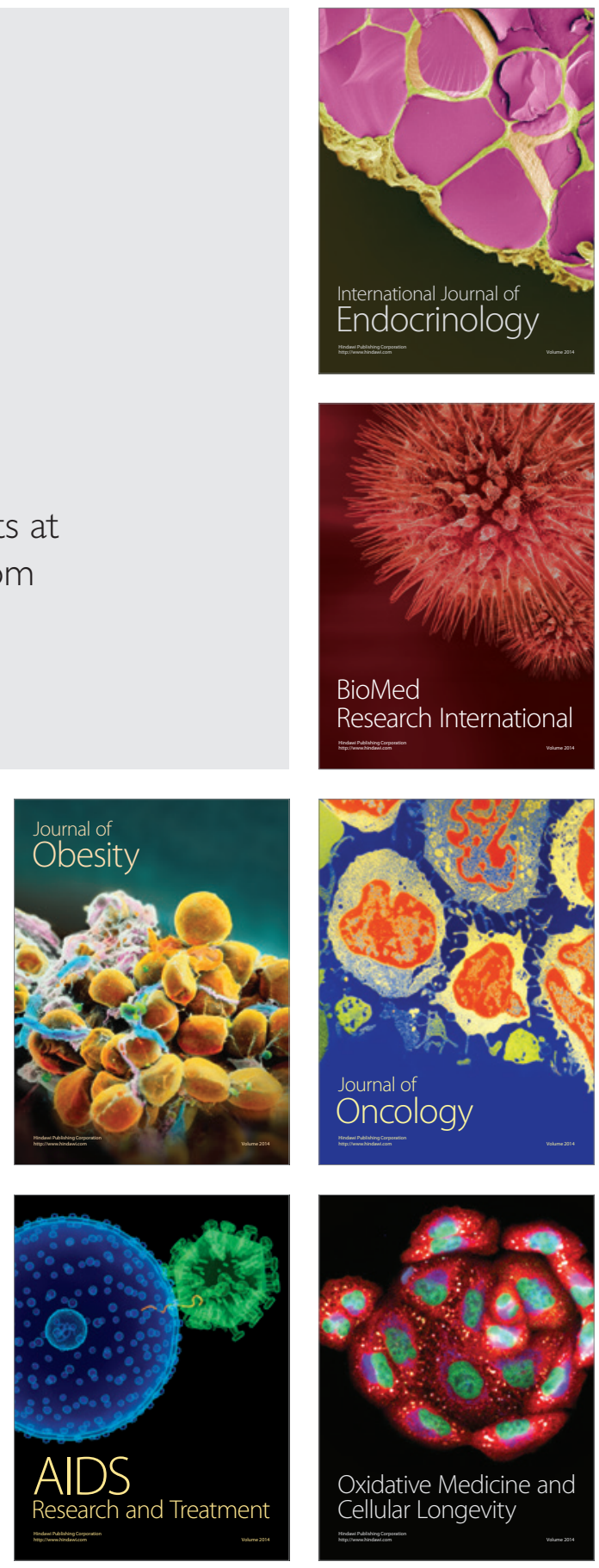